\title{
Detection of Wound Inflammations with Impedimetric Sensors
}

\author{
Anna Schröter ${ }^{1}$, Anja Walther ${ }^{2,3}$, Kerstin Fritzsche ${ }^{2}$, Dagmar Wersing ${ }^{3}$, Angela Rösen-Wolff ${ }^{2}$, Gerald \\ Gerlach $^{1}$ \\ 1 Solid-State Electronics Laboratory, Technische Universität Dresden, 01062 Dresden, Germany \\ 2 Department of Pediatrics, University Hospital Carl Gustav Carus, Fetscherstr. 74, 01307 Dresden \\ ${ }^{3}$ Institute of Materials Science, Technische Universität Dresden, 01062 Dresden, Germany
}

\begin{abstract}
In clinical practice a common possibility to monitor infections of wounds is to change the wound coverage in certain time intervals of 24 to 48 hours. This changing of dressing induces stress for the micro ambience of the wound. To support wound healing it makes sense to integrate a sensor into the bandage that detects an infection and, consequently, avoids stress factors generated by frequent changing of bandages. The wound environment changes during an inflammation process, e.g. temperature, $\mathrm{pH}$-value, composition and viscosity of the exudate. The challenge in designing sensors for this application is to find appropriate parameters that definitely indicate an infection. For this purpose it is reasonable to develop a multi-parametric determination.
\end{abstract}

\section{Keywords}

impedance spectroscopy, wound sensing, interdigitated electrodes, functional coating

\section{Medical background}

Neutrophil granulocytes belong to the main actors in acute inflammation processes during wound healing. As a part of the innate human immune system these cells react to germ contact by forming neutrophil extracellular traps (NETs). These NETs consist of ds DNA, histone proteins and several components of granula. NETs were first described as nanofibres with a diameter of 15 to $17 \mathrm{~nm}$ in 2004 [1]. They appear in 50 to $100 \mathrm{~nm}$ agglomerates as shown in figure 1. Neutrophil extracellular traps immobilize and kill germs.

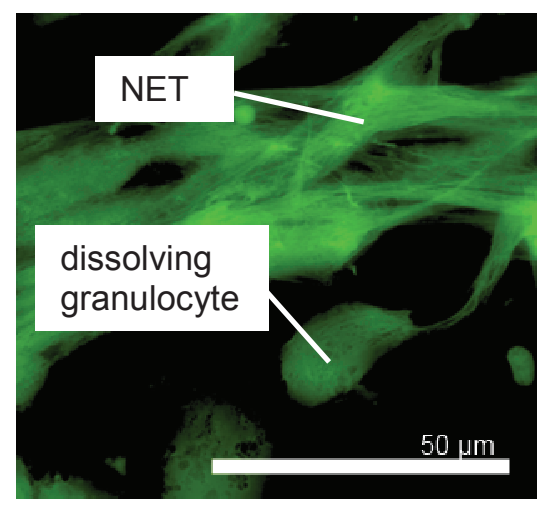

Figure 1: Fluorescence micrograph of NETs (stained with Sytox Green, 600x, by K. Fritsche)

\section{Sensor conception}

Normally, wound exudate is characterized by a low viscosity due to its origin from blood plasma. During inflammation viscosity rises when NETs are formed. The mean free path for dissolved ions and molecules changes by applying an electric field. Electrical impedance spectroscopy (EIS) records these changes as deviation in the complex impedance. Depending on structural properties several characteristic frequencies exist when these changes reach a maximum.

These frequencies can be detected by performing an EIS measurement on cells which form NETs in vitro. We determined the changes of electrical behavior by using commercial electrodes (Roche $x$ Celligence $\circledR$ ) connected to an impedance spectrometer (Inphaze). The electrodes were coated with Poly-L-Lysine to 
enable cell adhesion. A synthetic stimulans PMA (Phorbol 12-myristate 13-acetate) activated the granulocytes to form NETs.

\section{Impedance measurement results}

Structural changes can be deciphered when expressing impedance as a conduvtivity and a capacity in parallel. This is the most simple equivalent circuit. Results are shown in figure 2. Two frequency ranges are characteristic. At low frequencies capacitance changes drastically. In this range the system behaves like a capacitor. Capacitance is altered by formation of a NET biofilm. At higher frequencies of about $10 \mathrm{kHz}$ conductance of stimulated cells drops. This point is the inflection point where the setup gets conductive.
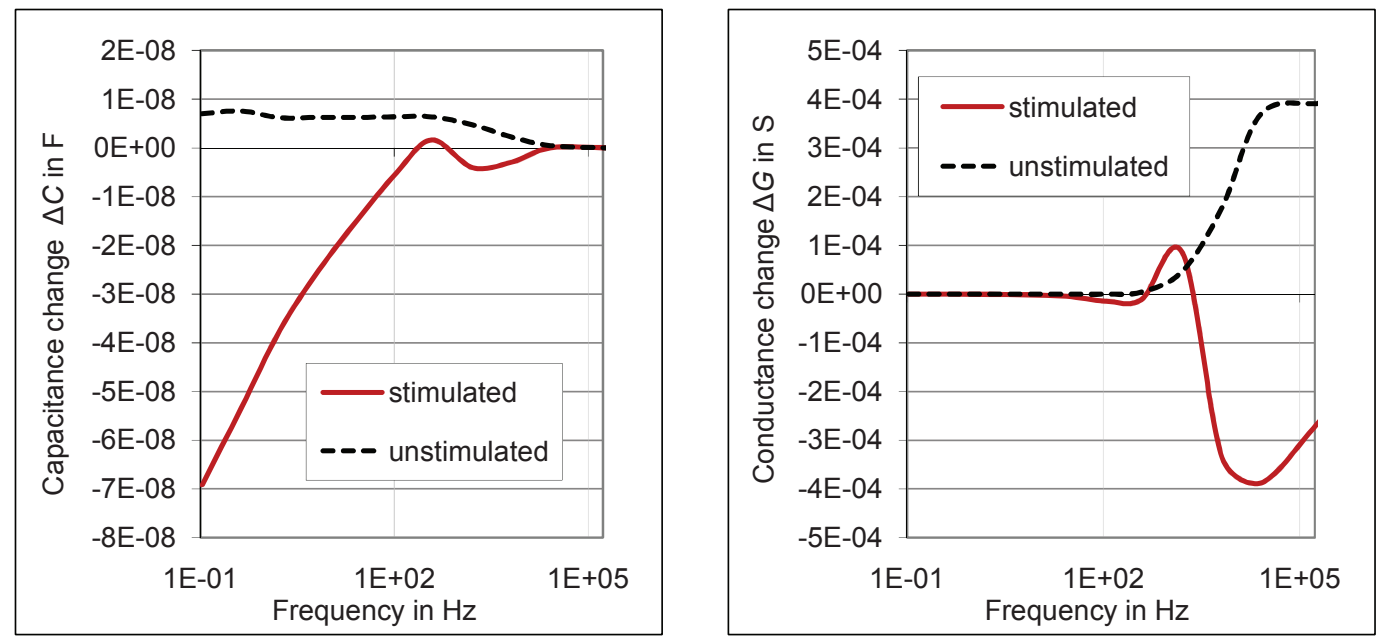

Figure 2: Capacitance and conductance change after 3 hours of PMA stimulation of granulocytes (10 measurements averaged). Unstimulated granulocytes are used as a control.

\section{Conclusions}

With the described setup, spectra of stimulated and unstimulated granulocytes were recorded. Conclusions about ideal excitation frequencies for capacitive and conductive measurements were drawn from the determined results. They can be used to design functional coated electrodes to enhance the measuring signal. These coatings can be made of biopolymers with different sensitivities which change their molecular structure in presence of special substances. To realize an ascertainment of several parameters the new concept contains a multi-area sensor. Each area has the capability to measure one quantity with impedimetric methods. This is especially useful for measurements in wet environments like a wound. Transfering this concept into an integrated setup enables identification of inflammation without opening the wound cover.

\section{Acknowledgements}

The authors thank the Bundesministerium für Bildung und Forschung for funding the project "ChiBSChip-basierte Biologie für die Sensorik" (project number 45952) within the framework of the "WKPotenzial" programme.

[1] Brinkmann et al., Science 303, 1532 (2004). 